\title{
Visualization of the Pollen Tube Cytoskeleton using Structured Illumination Fluorescence Microscopy
}

\author{
A. Geitmann, O. Gossot, and R. Zerzour
}

Institut de recherche en biologie végétale, Department of biological sciences, University of Montreal, 4101 rue Sherbrooke est, Montréal, Québec H1X 2B2, anja.geitmann@umontreal.ca

Pollen tubes have a highly anisotropic mode of growth in which the cytoskeleton has various different functions. Actin filaments are known to ensure the transport of vesicles from the Golgi apparatus to the apex. These vesicles provide the precursors necessary for the continuous formation of new cell wall and their rapid transport along actin arrays is thus pivotal for pollen tube elongation. However, studies have shown that the inhibition of actin polymerization stops pollen tube growth independently of the arrest of cytoplasmic streaming [1,2]. This suggests that actin could have a more direct implication in pollen tube elongation. Plant cell growth is dominated by an equilibrium between the pliability of the cell wall and the turgor pressure which creates forces that are considerably bigger than those potentially generated by actin polymerization. Therefore, actin is unlikely to be able to push forward the growing apex as is the case in the leading edge of migrating animal cells, but it could nevertheless have a mechanical function in apical growth. Microtubules on the other hand do not seem to be pivotal for pollen tube elongation proper, as application of microtubule depolymerizing agents do not stop growth. They might, however, be implicated in the directionality of growth or in the application of invasive forces.

To identify the putative structural roles of actin filaments and microtubules in pollen tube growth we needed to characterize the cytoskeletal configuration, in particular in the growing apex. While it is well known that both actin and microtubules are present in longitudinally oriented arrays in the distal part of the cell [3], the organisation in the apex is still a matter of discussion [2,4-6]. Next to an optimized fixation or good quality in vivo labeling techniques, the quality of the microscopic imaging is crucial for these filamentous structures. We used various fixation techniques and GFP label in combination with conventional fluorescence microscopy, confocal laser scanning microscopy and structured illumination fluorescence microscopy to analyze the spatial configuration of actin filaments and microtubules in Papaver rhoeas and Nicotiana tabacum pollen tubes.

Transformation of pollen tubes with ADF (actin depolymerizing factor)-GFP revealed that the actin cytoskeleton is very dynamic in growing pollen tubes. While observation of living pollen tubes is likely to show a cytoskeletal configuration that is the closest to reality, the dynamics of the cytoskeletal elements prevented high quality 3D imaging due to the time necessary to acquire complete z-stacks in both the confocal microscope and structured illumination. Chemical fixation on the other hand, albeit prone to artifacts, allowed us to produce excellent images which were close to configurations observed both in GFP labeled tubes and tubes fixed using rapid freeze fixation. These were characterized by a subapical actin fringe consisting of short, longitudinally arranged actin bundles in the cortical cytoplasm.

One of the fixation methods we tested relied on the fixing agent MBS (M-Maleimidobenzyoyl-Nhydroxysulphosuccinimide)-ester, a crosslinker which is known to stabilize the actin cytoskeleton. At high concentrations, this substance causes the appearance of highly crosslinked actin configurations. We observed that at low concentrations, however, the subapical actin fringe was prevented from moving forward while pollen tube growth continued for few minutes. We used this 
phenomenon to test several hypotheses for the putative structural function of the actin cytoskeleton in invasive pollen tube growth. We were able to show that the prominent subapical actin fringe seems to be involved in the control of the diameter of the cylindrical cell. Furthermore, partial inhibition of actin polymerization was able to reduce the pollen tube's capacity to invade a stiffened artificial medium, whereas the depolymerization of microtubules does not interfere with this important function of the cell.

\section{References}

[1] C.Y. Chen et al., The Plant Cell 14 (2002) 2175- 2190.

[2] L. Vidali et al., Mol Biol Cell 12 (2001) 2534-45.

[3] A. Geitmann \& A.M.C. Emons, J Microscopy 198 (2000) 218-245.

[4] I. Foissner et al., Protoplasma 2002 (2002) 1-15.

[5] A. Geitmann \& E. Parre, Sexual Plant Reproduction 17 (2004) 9-16.

[6] A. Lovy-Wheeler et al., Planta (Berl) 221 (2005) 95-104.

[7] This research was supported by grants from the Natural Sciences and Engineering Research Council of Canada (NSERC) and the Fonds Québécois de la Recherche sur la Nature et les Technologies (FQRNT).



FIG. 1. Papaver rhoeas pollen tubes labeled with fluorescent phalloidin. The subapical cortical fringe formed by actin filaments is a prominent feature in growing pollen tubes. Here, prior to formaldehyde fixation, tubes were treated with low concentrations of MBS-ester. This allowed the elongation of the tube while the fringe was inhibited from moving forward.

FIG. 2. Papaver rhoeas pollen tube labeled for mictrobubules using a tubulin-specific first antibody. In the distal region microtubules are arranged parallel to the longitudinal axis, whereas in the apex (right) shorter arrays are present in a less oriented configuration. The intensely labeled patch in the center is the generative cell which is rich in microtubules.

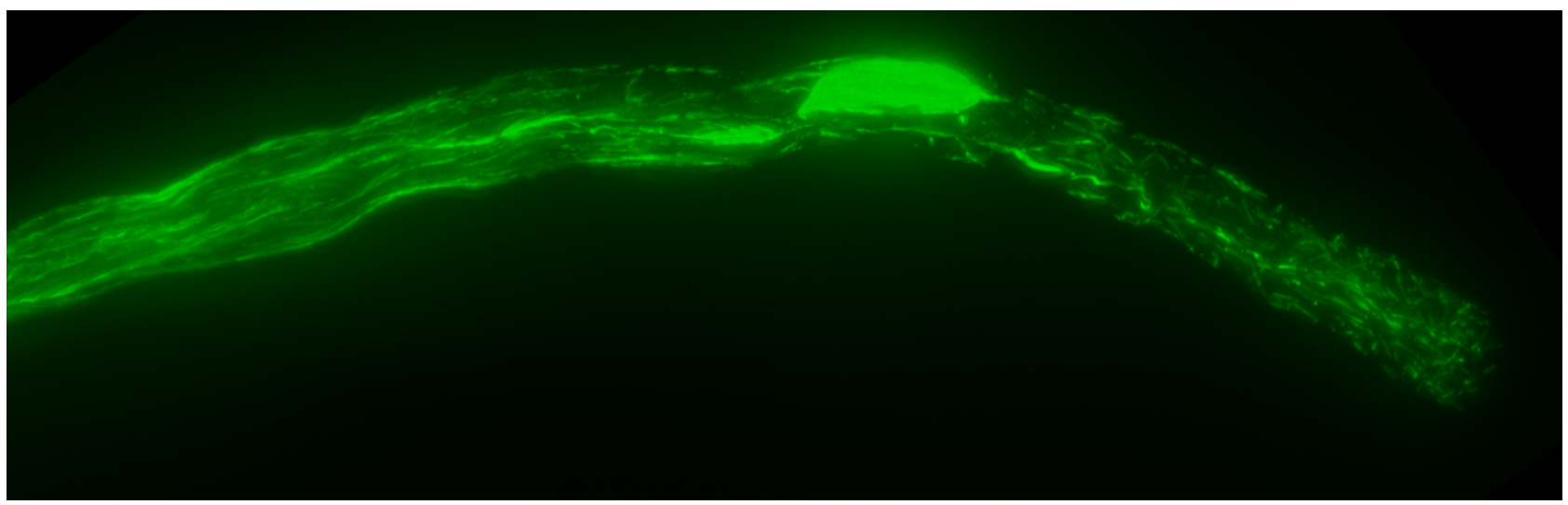

